\title{
Civilisations
}

Revue internationale d'anthropologie et de sciences

humaines

$63 \mid 2014$

L'Ancestralité revisitée

\section{La figure du fondateur comme incarnation $d u$} public

Mutations de l'ancestralité en contexte urbain

(Delta des Perles, Chine)

Anne-Christine Trémon

\section{OpenEdition}

\section{Journals}

Édition électronique

URL : http://journals.openedition.org/civilisations/3710

DOI : 10.4000/civilisations. 3710

ISSN : 2032-0442

\section{Éditeur}

Institut de sociologie de l'Université Libre de Bruxelles

\section{Édition imprimée}

Date de publication : 30 septembre 2014

Pagination : 123-142

ISSN : 0009-8140

\section{Référence électronique}

Anne-Christine Trémon, « La figure du fondateur comme incarnation du public », Civilisations [En ligne], 63 | 2014, mis en ligne le 30 septembre 2018, consulté le 20 avril 2019. URL : http:// journals.openedition.org/civilisations/3710; DOI : 10.4000/civilisations.3710 


\title{
La figure du fondateur comme incarnation du public
}

\author{
Mutations de l'ancestralité en contexte urbain
}

(Delta des Perles, Chine)

\author{
Anne-Christine TRÉMON
}

Résumé : Cet article se propose de revisiter l'ancestralité dans un ancien village situé dans la Zone économique spéciale (ZES) de Shenzhen, en Chine; créée presque ex-nihilo en 1980, celle-ci est devenue une mégalopole de plus de huit millions d'habitants. Il s'agit de montrer comment l'ancestralité s'est métamorphosée dans le contexte de l'urbanisation. Depuis la création de Shenzhen, le village quasi mono-lignager des Chen, fondé au $18^{\text {ème }}$ siècle, a vu sa population fortement augmenter. Désormais fondu dans l'agglomération urbaine, il n'existe plus administrativement comme village. Cet article montre comment les changements liés à l'urbanisation, et principalement la réforme foncière, se sont accompagnés d'une modification du rapport aux ancêtres, en se penchant plus particulièrement sur le statut de l'ancêtre fondateur du lignage-village et la place renforcée que celui-ci s'est vu conférer. Sa tombe, menacée de destruction par les plans d'urbanisation, a été préservée par la construction d'un mausolée qui la surplombe et qui sert également de cimetière public. Plus largement, la figure de l'ancêtre fondateur sert de porte-drapeau à l'action et à l'identité collective des membres de l'ancienne communauté villageoise. On assiste ainsi, en dépit de, mais aussi en raison même de la disparition $d u$ village mono-lignager, à une reformulation de l'ancestralité dans le nouveau cadre urbain. L'ancestralité a muté à travers un nouveau partage de l'équilibre entre "privé » et " public ».

Mots-clés : Chine, Shenzhen, ville, ancêtre, lignage, urbanisation, public.

\begin{abstract}
This article revisits 'ancestrality' in a former village of the Shenzhen Special Economic Zone in China. Shenzhen has been created almost ex nihilo in 1980 and has become a megalopolis that counts over eight million inhabitants. It is shown how ancestrality has changed in the context of urbanization. Since the creation of Shenzhen, the almost-single-lineage village of the Chen that was founded in the mid $18^{\text {th }}$ century has seen its population surge. It is now overwhelmed by the urban sprawl, and no longer exists administratively as a village. The article shows how changes brought about by urbanization, mainly the funeral reform, has generated a change in the relationship to ancestors. It focuses more particularly on the status of the founding ancestor and his enhanced role. When his grave was threatened to be destroyed, the lineage members protected it by building a mausoleum around it, a building that also serves as a public cemetery. Furthermore, the figure of the founding ancestor serves as a banner for the collective action and identity of the former village community members. There is a thus, in spite of, but also because of the disappearance of the former lineage-village, a reformulation of ancestrality in the new urban context. Ancestrality has mutated in the direction of a new balance between the 'private' and the 'public'.
\end{abstract}

Keywords: China, Shenzhen, city, ancestor, lineage, urbanization, public. 
$\mathrm{F}$ ort-les-Pins était jusqu'à récemment un village quasi mono-lignager emblématique du « complexe lignager-villageois » mis en évidence par Maurice Freedman (1958, 1966) pour la région du delta des Perles de la province du Guangdong. La dernière édition de la généalogie des Chen de Fort-les-Pins, parue en 2000, retrace l'histoire du lignage et détaille les liens reliant les habitants du village et leurs parents installés à Hong Kong ou à l'Outre-mer à l'ancêtre fondateur Chen Zhenneng 陈振能. La profondeur généalogique qui atteint, selon les branches, au maximum dix à douze générations, n'est pas très importante au regard d'autres lignages de la région ${ }^{1}$. Ceci tient à ce que les Chen de Fort-les-Pins appartiennent à la minorité ethnolinguistique hakka, formée au cours de plusieurs siècles de migration (Cohen 1996 ; Leong 1997). Dans le delta des Perles, les Hakkas étaient des nouveaux venus au regard de la population cantonaise plus anciennement installée. Venu du nord de la province du Guangdong, Zhenneng est le premier Chen à s'être installé en cet endroit au milieu du $18^{\text {ème }}$ siècle, et il est à ce titre l'ancêtre fondateur (shizu 始祖) du lignage des Chen et du village de Fort-les-Pins.

Dans ce texte, j'examinerai comment l'ancestralité a muté dans le contexte de l'urbanisation de ce village, en me penchant plus particulièrement sur le statut de l'ancêtre fondateur et la place renforcée que celui-ci s'est vu conférer. Fort-les-Pins n'existe plus aujourd'hui en tant que village. Les rizières qui en marquaient les limites ont été entièrement recouvertes d'usines, et les collines alentour sont masquées par des immeubles toujours plus hauts. Fort-les-Pins comptait à peine plus de 3000 habitants à la fin des années 1970 ; à présent sa population s'élève à quelques 60000 résidents dont plus de $95 \%$ sont des immigrants temporaires. En effet, ce sont les villages ruraux situés en périphérie de la zone urbaine centrale, entièrement planifiée, de Shenzhen qui ont accueilli l'essentiel des travailleurs migrants (Po 2012; Wang e.a. 2009: 959). Shenzhen, déclarée «zone économique spéciale » chinoise en 1980, est devenue la " première ville chinoise sans villages » au terme de l'opération d'urbanisation (chengshihua 城 市化), qui a supprimé en vagues successives plusieurs centaines de villages de la carte administrative. Fort-les-Pins a été touché par la dernière vague, en 2004. Le village en tant qu'entité autonome a disparu, étant converti en «communauté » (shequ 社区), la plus petite unité au sein de la hiérarchie urbaine. Fort-les-Pins est la traduction que j'ai choisi de donner de l'ancien nom du village, Song Yuan Xia (松元厦, Chung Yan Ha en hakka). Le village n'existe plus officiellement et la «communauté » ne porte plus ce nom.

Le statut résidentiel ( $h u k o u$ 户口) des habitants de l'ancien village, qui ne travaillent plus la terre depuis le début des années 1990, est passé de " paysan » à citadin, et leurs détenteurs ont été catégorisés comme « villageois d'origine » (yuancunmin 原村 民). À Fort-les-Pins, ils sont au nombre de 1441 , soit $2,4 \%$ de la population totale. $90 \%$ d'entre eux portent le nom Chen; le restant se distribuant en quelques patronymes minoritaires, principalement les Huang et les Ye. La communauté des villageois d'origine, laquelle comprend tant des Chen nés localement que des Chen rentrés de

1 Les travaux historiques sur le lignage (Ebrey 1986 ; Faure 1989) ont avancé qu'il est une invention culturelle historiquement située et dont la généralisation est liée à des évolutions politiques et économiques advenues sous les Ming (1368-1644). 
l'Outre-mer ${ }^{2}$ a considérablement bénéficié, au cours des trente dernières années, de la rente immobilière générée par le boom économique de Shenzhen. Aux revenus individuels qu'une majorité de villageois retire de la location de logements construits sur les lopins de terre distribués au début des années 1980 sont venus s'ajouter ceux de l'économie collective. En effet, ce qui distingue la catégorie des « villageois d'origine » est leur droit à détenir une action dans les sociétés qui gèrent les revenus immobiliers tirés de la rente d'usines bâties sur les anciennes terres collectives du village $^{3}$. À Fort-les-Pins, sept petites sociétés par action, correspondant aux anciennes brigades de production, gèrent des portions territoriales distinctes. Elles distribuent à leurs actionnaires des revenus annuels sous forme de dividendes, une assurance maladie ainsi que des pensions de retraite. Une huitième société œuvre à l'échelle de tout l'ancien village.

En théorie, l'urbanisation légale entraîne le transfert du droit d'usage sur les terres à l'État. À Shenzhen, le dispositif des sociétés par actions a assuré une transition relativement douce au regard de ce qui se produit ailleurs en Chine. En outre, la présence de lignages confère aux anciennes communautés villageoises une unité leur permettant de peser face aux autorités étatiques, en même temps que la persistance de cette forme d'économie collective contribue au maintien de la communauté lignagère. $90 \%$ des terres sont restées sous le contrôle des sociétés par actions, dont les dirigeants, élus par les assemblées des actionnaires, et qui sont également à la tête des comités de résidents (juweihui 居委会), sont tous des Chen. La collectivisation des terres effectuée au début de l'ère communiste a contribué à assurer une continuité en matière de gestion des terres lignagères, aujourd'hui gérées collectivement par la grande société par actions.

Le lignage subsiste également en tant que communauté dont les membres pratiquent collectivement des rituels à l'adresse de leur ancêtre commun. Ces pratiques cultuelles ont été interdites à l'avènement du régime communiste et de nombreux temples aux ancêtres ont été fermés ou reconvertis en écoles (c'est le cas de celui de l'ancêtre Zhenneng à Fort-les-Pins), voire détruits sous la Révolution culturelle. À Fort-les-Pins, la tablette (shenzhupai 神主牌) qui représente l'esprit de l'ancêtre fondateur Zhenneng, logée dans le temple ancestral, et la stèle tombale avaient été respectivement jetées dans un bassin et dans le fossé d'une porcherie. À partir de la fin des années 1950, le culte ancestral a été poursuivi à Hong Kong par des Chen qui avaient fui le village. La fondation Zhenneng (Zhenneng jijinhui振能基金会) a, pendant plusieurs décennies, financé le culte annuel rendu à l'ancêtre, pour les besoins duquel une nouvelle tablette avait été fabriquée sur place.

Bien que toujours considérés comme relevant de croyances superstitieuses et de formations sociales féodales, les cultes lignagers sont tolérés depuis l'entrée dans l'ère

2 Le lignage des Chen comme nombre d'autres lignages de la région, a longtemps été une véritable « filière d'émigration » (Watson 1975 : 5). Le recensement mené durant l'été 2010 à l'échelle de la « communauté » liste 1160 Chen à l'Outre-mer (en Europe, en Asie, dans le Pacifique et en Amérique du Nord et centrale) et 1429 à Hong Kong. Le nombre de Chen à l'Outre-mer est bien supérieur en réalité, le chiffre ne donne une indication que des relations familiales effectivement maintenues.

3 Suivant un dispositif appliqué dans l'ensemble du Guangdong, ces sociétés coopératives par actions (gufen hezuo gongsi股份合作公司) ont été conçues de manière à assurer la transition vers « l'économie socialiste de marché »". 
dite des « réformes et de l'ouverture » qui a débuté en 1978. Les activités cultuelles ont repris - comme les activités religieuses en général ailleurs en Chine - au cours des années 1980 et 1990. À Fort-les-Pins, les rites biannuels rendus à l'ancêtre Zhenneng, l'un au temple ancestral, l'autre sur sa tombe, ont repris dès 1981. Cette année là, la tombe de Zhenneng a été re-cimentée et une nouvelle stèle tombale y a été apposée ; la tablette de l'ancêtre a été repêchée et réinstallée au fond du temple ancestral, avant d'être remplacée, quelques années plus tard, par une tablette neuve. Ces réparations et restaurations ont été largement financées par les Chen de Hong Kong et d'Outremer, de même que toute une série d'opérations de construction ou de reconstruction : l'école primaire Zhenneng, en 1987, un portique d'entrée au site où se trouve la tombe de Zhenneng, en 1995, l'immeuble hébergeant le foyer d'activités pour les personnes âgées, en 1997. Ce dernier, «l'immeuble de la culture Zhenneng » (Zhenneng wenhua dalou 振能文化大楼) a été rasé et reconstruit avec quelques étages supplémentaires loués à des migrants en 2004. Il héberge depuis lors la fondation Zhenneng, rapatriée de Hong Kong.

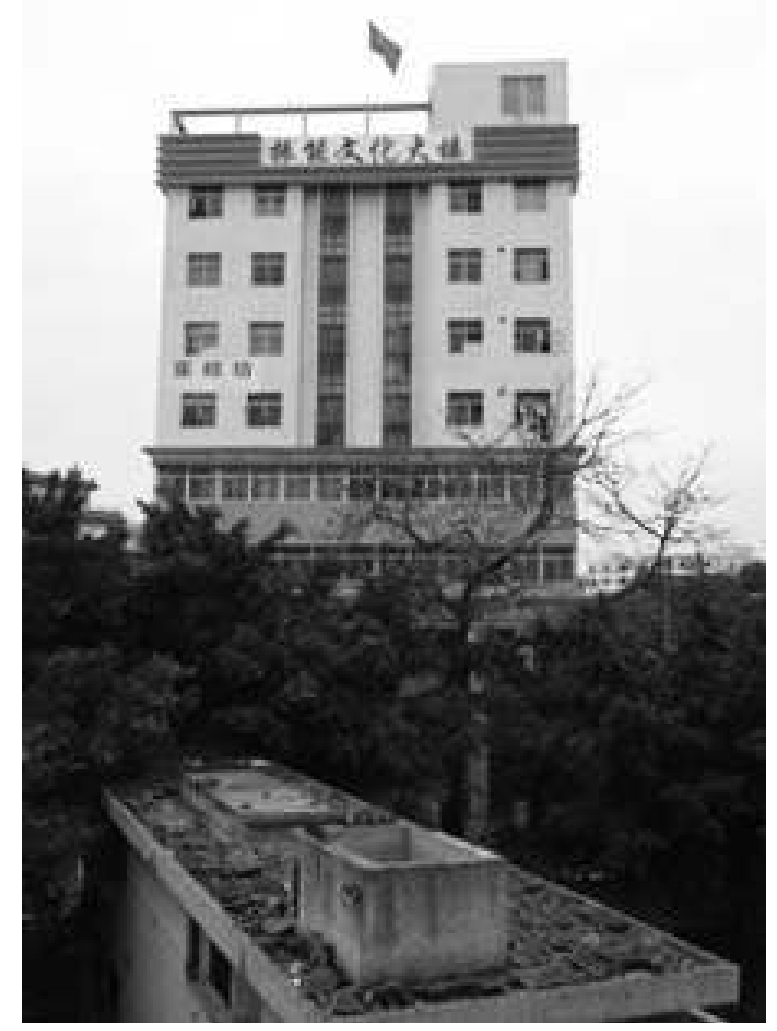

Immeuble de la culture Zhenneng, siège de la fondation lignagère. (C) Anne-Christine Trémon, 2013. 
Certains chercheurs ont considéré le renouveau de la religion populaire comme une réaffirmation de mémoires historiques (Jing 1996) et de moralités collectives (Yang 2000, 2004) pré-révolutionnaires, alors que d'autres ont avancé que les paysans sont des « semi-croyants » (Chau 2011) et que les temples sont des « hybrides » mi-modernes mi-traditionnels (Flower 2004). Siu (1989) a quant à elle avancé que les rites collectifs relèvent davantage d'un égalitarisme paysan témoignant de la pénétration profonde des idéaux de l'état socialiste dans la société locale que d'une véritable adhésion à la signification de ces rites. Des interprétations divergentes ont également été données de l'économie rituelle qui accompagne la revitalisation de ces cultes. Alors que Yang (2000) la considère comme une destruction ostentatoire des richesses produites par la nouvelle économie capitaliste, destinée à protéger la sphère morale de la parenté et de la communauté, Brandstädter (2013) y voit plutôt un contraste entre l'individualisme du secteur capitaliste privé et une économie collective qui soutient la recherche du bien public. Les matériaux que j'ai récoltés jusqu'à présent vont dans ce sens.

Mes recherches à Fort-les-Pins portent moins sur le renouveau religieux en luimême (d'autant que, nous le verrons, l'édification du mausolée auquel je m'intéresse dans ce texte n'a pas été dictée par un souci de revitalisation) que sur les relations entre membres du lignage local et leurs parents dans la diaspora. Les participations financières de ces derniers sont présentées comme contribuant au bien public. Plus généralement, les projets collectifs menés à Fort-les-Pins, dont les infrastructures et rites lignagers mais aussi tout ce qui participe au développement économique de l'ancien village, le sont au nom du « bien public » (gongyi 公益) et sont présentées comme relevant de la «cause publique » ou des « affaires publiques » (gongyi shiye 公益事业). Je considère l'inscription du culte lignager dans ce registre du bien public comme le double produit d'une longue tradition historique de l'investissement lignager dans les « affaires publiques », telles que les écoles et les œuvres philanthropiques, et de l'appropriation et la perpétuation, par les communautés paysannes, dans le contexte de l'ère des réformes, du discours révolutionnaire de l'ère maoïste.

Ceci vaut d'autant plus qu'à Fort-les-Pins comme ailleurs, les cadres et dirigeants locaux sont des membres du lignage qui siègent au conseil de la fondation Zhenneng. Ils sont ainsi soumis à la pression morale des membres de la communauté lignagère et villageoise tout en retirant de l'influence politique de leur engagement dans les activités culturelles. Ceci est bien entendu une des raisons expliquant la tolérance vis-à-vis de la reprise des cultes lignagers. L'autre raison tient à la participation des parents installés à Hong Kong et à l'Outre-mer, dont il s'agissait, au moins dans les premières décennies de l'ouverture, d'attirer les investissements. En effet, les Chinois de la diaspora ont joué un rôle primordial dans la restauration des temples et des activités cultuelles dans les provinces côtières qui ont été les premières à s'ouvrir aux capitaux étrangers. Alors que dans la province du Fujian, ce sont les temples et les rituels aux divinités locales qui ont été rétablis en priorité (Dean 2003 : 37), menant John Lagerwey à affirmer que les lignages ne constituent plus une unité sociale fondamentale (1997:5), dans le delta des Perles, c'est le culte lignager qui domine, et l'absence, à Fort-les-Pins, de temple autre qu'ancestral, ne constitue pas une exception. Dans le débat entre ceux qui affirment que les lignages n'existent plus en Chine aujourd'hui dès lors qu'ils ne peuvent plus prendre la forme des grandes corporations propriétaires foncières d'avant 1949 et ceux qui montrent leur résurgence en l'absence même de propriété immobilière à travers la 
restauration des temples aux ancêtres et la recompilation des généalogies (Yang 2000 : 487), la situation de Shenzhen donne raison aux seconds et ôte leurs arguments aux premiers : on constate ici que la collectivisation des terres sous Mao n'a pas détruit les communautés lignagères et que le maintien des collectifs dans l'ère des réformes en favorise la perpétuation.

Les travaux qui ont abordé l'ancestralité - le statut ontologique des ancêtres et les modes de relation qu'entretiennent avec eux les vivants - dans le monde chinois ont examiné le statut respectif des ancêtres, des dieux et des fantômes (Ahern 1971; Jordan 1972; Harrell 1974; Wolf 1974). Bien moins nombreux sont ceux qui ont interrogé le statut particulier de l'ancêtre fondateur. Une des rares tentatives opérées en ce sens est celle d'Allen Chun (1996), qui cherche à expliquer la nature des lignages chinois en référence à des notions sous-jacentes au culte ancestral. Il isole comme fondamental le concept d'ancêtre fondateur (shizu) qui serait selon lui apparu aux $17^{\text {ème }}$ et $18^{\text {ème }}$ siècles (1996 : 436). Son argumentation n'est cependant pas très convaincante et la manière dont il s'est exclusivement appuyé sur les écrits des idéologues a été très critiquée ${ }^{4}$.

C'est à partir de matériaux ethnographiques contemporains que je tâcherai de comprendre le statut de l'ancêtre fondateur et la nature du lien entretenu avec lui par les anciens villageois Chen de Fort-les-Pins. Si sa focalisation fonctionnaliste sur les relations entre stratification sociale et segmentation généalogique et son manque d'attention aux conceptions sous-jacentes au culte ancestral lui ont été reprochés, Maurice Freedman avait pourtant proposé une réflexion sur le statut des ancêtres à travers une réflexion sur la tension entre culte individuel des ancêtres immédiats et culte collectif de l'ancêtre commun. C'est cette réflexion, qu'il n'a pas menée à son terme, que je souhaiterais poursuivre ici en montrant comment l'ancestralité a muté à travers un nouveau partage de l'équilibre entre « privé » et « individuel », d'une part, et « collectif » et « public » d'autre part.

Les matériaux sur lesquels je m'appuie ont été recueillis durant quatre séjours dans le village (août 2011, juillet 2012, mars et octobre 2013) au cours desquels j'ai, entre autres, cherché à retracer le processus de construction d'un mausolée autour de la tombe de l'ancêtre fondateur Zhenneng'. Durant les recherches que j'avais menées précédemment auprès des Chinois en Polynésie française, les Chen originaires de Fortles-Pins m'avaient parlé de ce qu'ils appelaient le « mausolée » (je leur emprunte donc cette traduction du terme lingyuan 陵园) qui venait d'être construit, en 2000, et auquel ils avaient contribué financièrement. Alors que, pour cette raison, je concevais ce projet comme s'inscrivant dans la suite logique de l'ensemble des activités de renouveau lignager qui avaient eu lieu depuis le début des années 1980, j'ai découvert qu'il s'agissait en réalité non pas d'une entreprise de revitalisation, mais d'une opération de sauvetage liée à la réforme funéraire conduite à Shenzhen.

En retraçant cette opération de sauvetage, je montrerai comment elle a conduit à une mutation de l'ancestralité dans le sens d'une accentuation de la place de l'ancêtre

4 Par les commentateurs de son article paru dans Current Anthropology.

5 Au cours de ces séjours, j'ai observé les deux rites annuels, mené des entretiens et eu de nombreuses conversations informelles avec des habitants de Fort-les-Pins ou des membres du lignage résidant ailleurs et revenus pour les rituels. L'entrée sur le terrain a été facilitée par le fait d'y avoir été introduite par un Chen de Polynésie et par la connaissance préalable de la généalogie. 
fondateur en raison du principe d'unification dont celui-ci est le vecteur. Elle a consisté en une entreprise de « publicisation », transformant la tombe de Zhenneng en un lieu public et le nom de Zhenneng en emblème du bien public. Je soulignerai dans un premier temps comment les efforts déployés par les Chen de Fort-les-Pins pour sauvegarder la tombe de leur ancêtre fondateur et loger leurs ancêtres exhumés révèlent en creux leurs conceptions de l'ancestralité. Dans la section suivante, je montrerai en quoi la sauvegarde de la tombe de l'ancêtre fondateur n'avait rien de naturel ni d'évident, dès lors qu'il supposait de mettre en marche une mobilisation collective là où chacun pensait avant tout à ses ancêtres les plus proches. Ceci permettra, dans un dernier temps, d'apprécier à sa juste mesure le statut particulier de l'ancêtre fondateur et de mettre en relief ses propriétés d' " individu collectif» transcendant les intérêts individuels.

\section{Les ancêtres menacés de destruction}

Les Chen de Fort-les-Pins pratiquent les deux types de culte distingués par Freedman (1970 : 167-168), lignager et domestique. Le culte lignager est effectué deux fois l'an, sur des sites différents : au temple de l'ancêtre Zhenneng, le jour de son anniversaire au vingtième jour du neuvième mois lunaire (vers octobre) et sur sa tombe, le premier jour de Chunfen 春分 (la quatrième période solaire dans le calendrier chinois, débutant le 20 mars dans le calendrier grégorien). Le culte domestique, adressé aux ancêtres les plus proches, était effectué, jusqu'à récemment, sur les tombes. Dans le temple lignager, on trouve une seule tablette dédiée à l'ancêtre fondateur ainsi qu'à son épouse. Cela correspond à ce qui a été souvent observé pour les Hakkas du Guangdong (Aijmer 1967 : 57 ; Cohen 1969 : 170), à savoir que leur culte lignager se distingue de celui des Cantonais, qui disposent des tablettes pour chacun de leurs ancêtres. C'est le culte rendu sur les tombes qui différenciait individuellement les ancêtres de chaque génération, lors de la fête de Qingming, quinze jours après Chunfen. Ceci vaut toujours à présent que les tombes ont été détruites et que le culte est rendu devant les urnes contenant les cendres des ancêtres, au mausolée.

L'idée de construire un mausolée autour de la tombe de l'ancêtre Zhenneng a vu le jour quelques mois après que la municipalité de Shenzhen ait annoncé la « réforme funéraire », binzang gaige 殡葬改革, en mars 1997, procédant à une mise en application stricte et rapide des nouvelles régulations nationales édictées par le Conseil d'État. Cette réforme est en réalité à l'œuvre depuis l'arrivée au pouvoir du Parti communiste chinois, lequel promeut la crémation et la simplicité des funérailles. Cependant, la réforme s'est faite par à-coups, variant dans le temps en fonction des priorités politiques. Le principal résultat des réformes déployées sous l'ère maoïste a été la ligne de séparation tracée entre les pratiques funéraires urbaines et rurales, la crémation s'étant surtout généralisée en ville (Whyte 1998 ; Johnson 2011). L'accroissement de la population urbaine et le développement économique consommateur d'espace dictent à cet égard l'adoption des mesures préconisées à partir de 1997.

Plus récemment, les résistances rencontrées par la réforme dans le district de Zhoukou au Henan en 2012 et les protestations nationales auxquelles les événements qui s'y sont produits ont conduit à une modification apportée aux " stipulations nationales concernant l'enterrement » et notamment à l'article 20 qui affirmait qu'en 
cas de résistance, il pouvait être recouru à la force ${ }^{6}$. À Shenzhen, les opérations de déterrement et de crémation ont été menées tambour battant, quinze ans plus tôt. Il n'est pas étonnant que la réforme ait trouvé son terrain d'application le plus rapide dans les villes en pleine expansion, où il s'agissait de transformer ceux qui étaient catégorisés comme «paysans » en citadins modernes. C'est donc dans le contexte de l'urbanisation à marche forcée qu'il faut comprendre la manière drastique dont la réforme nationale y a été appliquée. À Shenzhen, il a été décidé d'atteindre dans les meilleurs délais un taux de $100 \%$ de crémations en prohibant désormais tout enterrement. Les autorités municipales sont allées très loin en ce qu'elles ont ordonné de déterrer tous les restes des défunts, de les brûler, et d'en disperser les cendres ou de les placer dans des cimetières publiquement accrédités. Dans le village de Fort-les-Pins, une équipe gouvernementale est venue durant l'année 1998 pour procéder à l'exhumation ; les villageois étaient menacés, s'ils s'y opposaient, ou s'ils cherchaient à cacher les ossements ${ }^{7}$, d'être privés de leurs parts dans les sociétés par action.

Je voudrais souligner la violence de la réforme funéraire dans sa conception même. Elle est énoncée au nom de la construction d'une " civilisation socialiste spirituelle » (shehuizhuyi jingshenwenming 社会主义 精神文明). Son but explicite est de réformer les coutumes funéraires (sangzang xisu丧葬习俗) en éliminant les « activités superstitieuses » (mixin huodong 迷信活动) ${ }^{8}$. Elle s'oppose frontalement aux pratiques de l'enterrement guidées par le principe de transformation des morts en ancêtres. Tout parent décédé doit recevoir un minimum de soins rituels, et s'il n'a qu'un descendant, celui-ci aura l'obligation de s'occuper de lui. Car un défunt non ancestralisé risquerait de se transformer en gui, esprit malfaisant assoiffé de vengeance (Ahern 1973 : 125 ; Baptandier 2001 : 15). Pour éviter qu'un mort revienne hanter et nuire aux vivants, il faut le transformer en ancêtre, et pour cela s'assurer qu'il soit bien enterré et bénéficie de tout ce dont il a besoin au royaume des morts.

Le culte des ancêtres constitue à lui-même sa propre fin; il a pour but de fabriquer des ancêtres et de les maintenir dans ce statut. On leur sacrifie de la nourriture et de la boisson, et on leur transmet, en les brûlant, toutes sortes d'objets en papier - maison, vêtements, téléphone portable, passeport - mais surtout d'importantes sommes de papier monnaie afin de s'acquitter de la dette contractée auprès d'eux (Gates 1987 : 268-269). En outre, on s'attend à ce qu'en retour, l'ancêtre prodigue des bienfaits à ses descendants - prospérité, descendance nombreuse, etc. Pour cela, il est important d'enterrer un parent défunt dans un endroit propice. La géomancie (fengshui 风水) exerce un effet bénéfique par le medium des ossements. La très grande dispersion des

6 Cf. l'article posté par un certain Yuan Gang, fort probablement un cadre du parti, pour défendre la réforme funéraire suite au mouvement de révolte. Blog gongshiwang 共识网, 3 décembre 2012, <http:// www.21ccom.net/articles/zgyj/ggcx/article_2012120372170.html $\geq$

7 Dans ce village hakka, on pratiquait jusque là le double enterrement ; les os étaient déterrés quelques années après l'enterrement et placés dans de grandes jarres elles-mêmes placées dans des tombes (Ahern 1971; Freedman 1966 : 118-154 ; Watson 1988 : 113). Watson et al. (1982: 112-5) a montré que cette pratique est liée à la peur de l'influence polluante des morts, et plus particulièrement de la chair. Ceci pourrait expliquer l'acceptation de la crémation imposée par les autorités ou tout au moins, l'absence de révolte contre cette imposition (Johnson 2011 : 128). 
tombes, que l'on trouvait partout autour des villages, à flanc de collines, résulte de ce que les parents des défunts recherchent le site géomantique idéal pour leurs défunts proches. Libérer les terrains occupés par les tombes est un but explicite de la réforme funéraire. Aussi sa rapidité d'exécution à Shenzhen doit-elle se comprendre au regard de l'urbanisation accélérée de cette zone, où la pression foncière devenait de plus en plus importante. À Fort-les-Pins, elle a coïncidé avec la planification d'une route qui devait rejoindre le terrain de golf récemment aménagé au nord-est de l'ancien village, et passer pile à l'endroit où se situe la tombe de l'ancêtre fondateur Zhenneng.

L'édification du mausolée a permis d'annuler ce projet routier mais avant tout de protéger les ossements de Zhenneng. En effet, le mausolée, dont la construction a été planifiée dès la fin de l'année 1997 et achevée en 2000, contient la tombe de Zhenneng dans sa partie centrale, ainsi que les urnes des ancêtres déterrés et ayant fait l'objet d'une crémation dans les ailes du bâtiment qui flanquent l'espace tombal. Les portes du milieu peuvent s'ouvrir sur toute la largeur de la tombe de telle façon que l'on peut l'embrasser du regard comme si elle était à découvert. La tombe, en forme d'omega, est surplombée d'un dôme, et entourée, de chaque côté, d'un escalier conduisant aux deux étages supérieurs. Chaque aile est partitionnée en espaces qui ont été alloués à chacun des ancêtres de la cinquième génération, espaces délimités par des cloisons recouvertes d'étagères où sont rangées les urnes de leurs descendants.

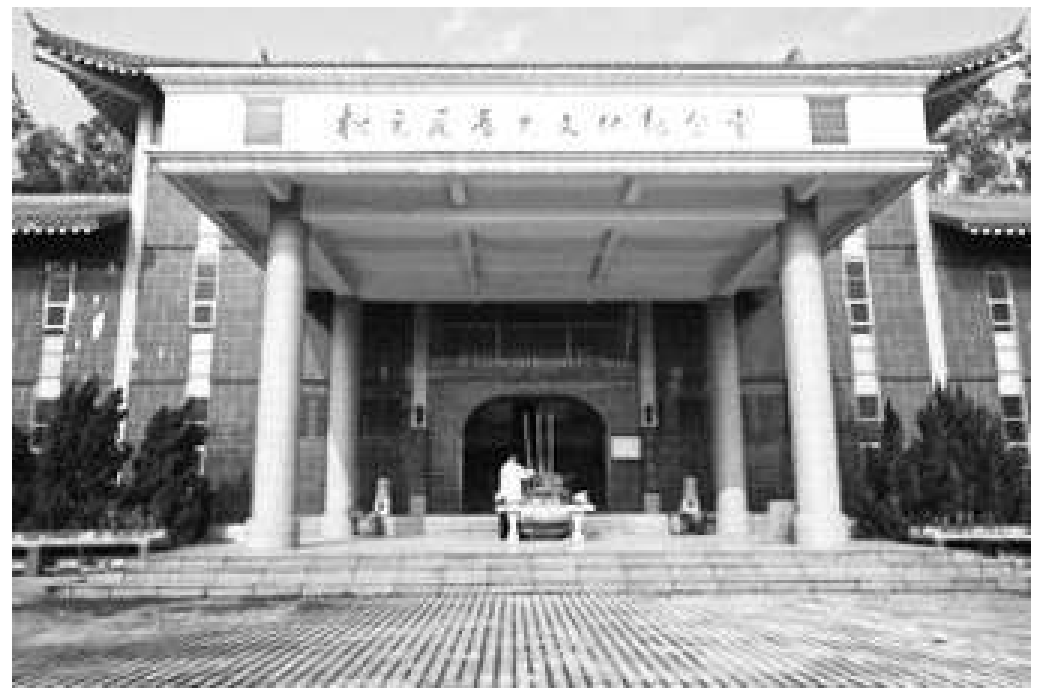

Le mausolée.

(c) Anne-Christine Trémon, 2013.

De plus, plusieurs espaces sont dédiés à des non-Chen ; les principaux lignages minoritaires du village, les Huang et les Ye, ont reçu un espace pour leurs ancêtres qu'ils ont été, au même titre que les Chen, sommés de déterrer et de crémer. Cette présence de non-Chen dans le mausolée est présentée par certains villageois comme un acte nécessaire de générosité vis-à-vis de leurs voisins, mais aussi comme une garantie 
de la légalité de leur entreprise, puisqu'elle confère de ce fait, au mausolée, un caractère public. Or c'était là tout le but de l'entreprise : échapper à l'illégalité en plaçant les urnes des ancêtres dans un espace officiellement accrédité, créé de toutes pièces à cette occasion.

L'action entreprise pour contourner les effets de cette réforme souligne l'importance revêtue par le substrat matériel, les ossements, sans lequel il ne peut y avoir de continuité du lignage. Dans la Chine ancienne, mais cela vaut pour aujourd'hui, « une manière certaine de détruire une lignée en châtiment d'un crime de l'un de ses membres, sans mettre à mort l'ensemble des parents, consistait à ouvrir les tombes et à sortir, en les dispersant, les ossements des parents défunts »(Baptandier 2012 : 289). Pis encore, la crémation ou la pulvérisation des restes était le meilleur moyen d'annihiler définitivement un ennemi (Lewis 2006 : 59-60). Cette remarque de Lewis s'appuie sur la révision qui est intervenue de l'interprétation sinologique longtemps dominante selon laquelle après la mort les âmes hun et po se sépareraient (les premières ascendant au ciel et les secondes retournant à la terre). Les recherches menées par Brashier sur les pratiques funéraires Han, basées sur l'archéologie des tombes, tendent à montrer que ces deux âmes sont inséparables et conçues comme résidant, l'une et l'autre, dans les ossements, le bon enterrement devant assurer leur rétention dans la tombe (Lewis $2006: 51)$.

Les villageois de Fort-les-Pins n'entrent pas dans ce type de considérations lorsqu'ils racontent la construction du mausolée. La sauvegarde de la tombe de Zhenneng est présentée comme un impératif catégorique, une nécessité si évidente qu'elle n'a pas à être justifiée (mais qui ne pourrait de toute façon pas l'être sous peine d'être taxé de superstition). Les personnes à qui je demandais comment le projet du mausolée avait émergé répondaient en faisant référence au résultat et non à la motivation ; on me répondait par exemple que s'il n'avait pas été construit, les restes (haigu 骸骨) de l'ancêtre n'auraient pas été en sécurité (baobuwen 保不稳). L'importance de la place de la tombe dans le culte ancestral en Chine méridionale est démontrée, dans ce cas contemporain, par les efforts considérables, et à la limite de la légalité, qui ont été déployés par les membres du lignage Chen pour protéger les ossements de leur ancêtre fondateur. Ceux de son épouse ont également été préservés, puisqu'elle était enterrée dans un site devenu, en 1995, partie de l'immense golf voisin et avait de ce fait échappé à la menace jusqu'à ce que la construction de villas de luxe dans ce complexe de loisirs impose son exhumation. Le jour de l'équinoxe de printemps, les portes du mausolée sont maintenues grandes ouvertes et l'urne contenant les ossements de l'épouse placée devant la tombe de Zhenneng, de même que les urnes contenant les cendres des trois fils et de leurs épouses.

Si la tablette peut faire symbole, et devenir le support d'un culte ancestral délocalisé à Hong Kong, donc déterritorialisé, elle ne peut se substituer aux restes du corps. Sans le point de référence territorial qu'est la tombe, il n'aurait pu y avoir délocalisation du culte. Les ossements, en tant qu'expression pérenne de la personne - ce qui en reste pour l'éternité - sont une condition sine qua non du culte ancestral dans son ensemble : sans restes de l'ancêtre, il n'y a plus d'ancêtre. Étant la partie immuable de l'ancêtre, les ossements sont un gage de son existence continuée après la mort et donc de sa présence perpétuelle, mais ils sont aussi le gage de la reproduction, et de la perpétuation du corps social lignager. Bien enterrés, les ossements sont censés agir au bénéfice 
des descendants, leur assurant prospérité et descendance si possible nombreuse, au minimum continuée.

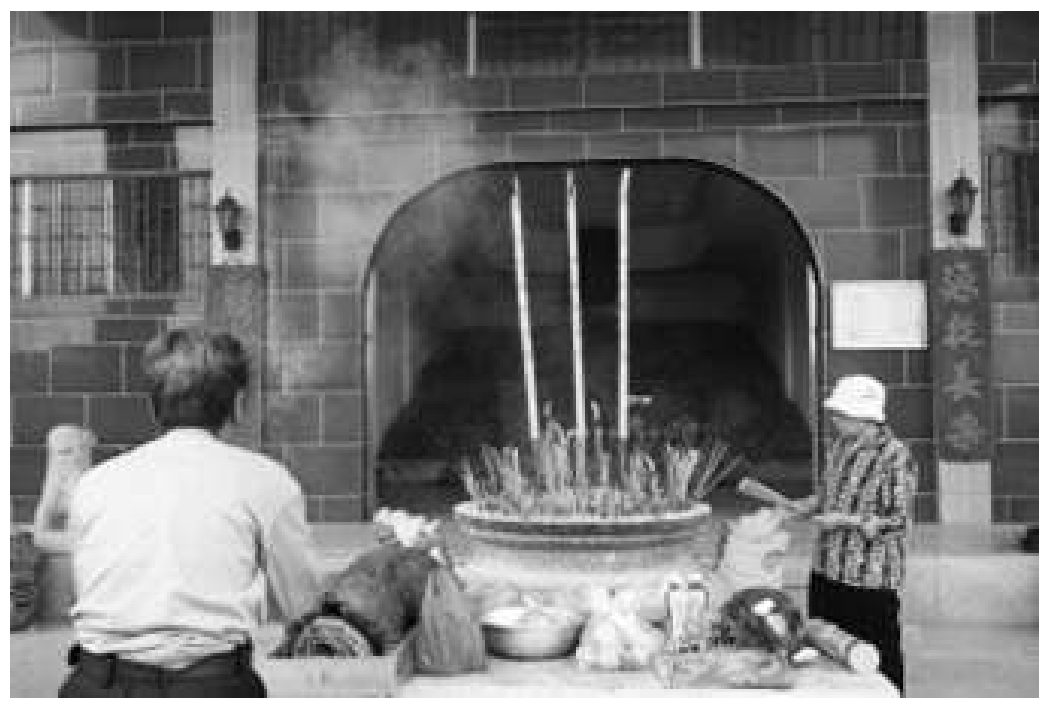

Sacrifice à Zhenneng, Chunfen (équinoxe de printemps), 20 mars 2013.

(c) Anne-Christine Trémon.

\section{L'ancêtre commun et les ancêtres proches}

Arrêtons-nous un instant à la déclaration suivante, émanant de l'un des dirigeants du village, Chunhua (elle est très ressemblante à celles que j'ai pu recueillir auprès d'autres villageois impliqués dans l'affaire) :

Durant cette réforme funéraire, nous avons pensé à notre ancêtre Chen, l'ancêtre fondateur de notre lignage (Chenshi zongzu de taigong 陈氏宗族的太公), ancêtre Zhenneng qui s'est installé ici et a fondé Fort-les-Pins ; aujourd'hui il est enterré dans ce mausolée, nous avons pensé que nous devions protéger ses ossements (haiti 骸体). Nous ne devons pas laisser des gens l'exhumer. Nous ne pouvions pas autoriser une chose pareille. Alors nous avons pensé à la chose suivante : construire un bâtiment audessus de sa tombe, de façon à protéger les ossements de l'ancêtre et mettre ensemble tout le village sans séparer le lignage (jiang quancun bufen xingshi 将全村不分姓氏), et alors nous avons déterré et brûlé tous les os des ancêtres du village, nous les avons placés dans des urnes funéraires et les avons rangées là, voilà comment cela c'est fait.

Cette déclaration est intéressante en ce qu'elle incite à ne pas s'en tenir uniquement à la nécessité de protéger l'ancêtre et à voir dans la possibilité de stockage des urnes obtenu par la construction du mausolée un simple corollaire de l'opération de sauvegarde. Elle montre bien que le but du projet était double, et que le second objectif était tout aussi (sinon plus) important que le premier. Trouver un espace de stockage des urnes de tous les ancêtres déterrés n'était pas simplement un avantage supplémentaire 
offert par le mausolée une fois construit. Car toute aussi grave que la destruction de la tombe de Zhenneng était la menace de dispersion des cendres des ancêtres déterrés.

Les Chen de Fort-les-Pins soulignent que ce qu'ils voulaient éviter à tout prix était la dispersion des cendres de leurs ancêtres (cette menace ayant dans leurs propos le même statut logique que celle pesant sur la tombe de Zhenneng, quelque chose de l'ordre de l'inadmissible). C'était le même terme qui revenait pour désigner la menace qui avait pesé, fensan 分散, séparation, dispersion. La récurrence de cette expression me surprit d'abord car je l'entendais par ailleurs en référence aux tombes (lorsque je demandais où celles-ci se trouvaient), dispersées tout autour du village. Mais il est clair que la dispersion n'était pas la même ; dans un cas, les ancêtres demeurés enterrés dans les limites de leurs villages, alors que dans le second, après crémation, ils menaçaient de se voir placés dans un cimetière à l'extérieur du village, ou pire, placés dans les maisons de leurs descendants - à Fort-les-Pins -, mais ailleurs aussi. L'importance d'éviter cette dispersion se perçoit dans l'expression utilisée dans la citation des propos de Chunhua, ci-dessus : " mettre ensemble tout le village sans séparer le lignage ».

Le risque était d'autant plus grand que chacun, dans un premier temps, ne pensa qu'à ses ancêtres directs. S'il est rétrospectivement évident qu'il fallait absolument protéger les ossements de l'ancêtre fondateur, il ne faudrait pas prendre pour argent comptant les rationalisations à posteriori de mes interlocuteurs. Dans l'urgence du moment, la réaction de chacun fut d'abord de chercher où loger " ses propres ancêtres ", selon l'expression courante (ziji de zuxian 自己的祖先). De plus, la mobilisation collective que nécessitait une entreprise d'une telle envergure n'allait pas du tout de soi, surtout dès lors qu'il s'agissait de contourner les règlements ou de s'aventurer dans une zone grise, à la limite de la légalité.

Pour résumer le processus, c'est le retour au village, dans le cours de l'année 1997, d'un membre du lignage et ancien cadre gouvernemental très haut placé, Ganwan - il avait terminé sa carrière comme vice-directeur de l'aéroport de Shenzhen - qui a permis de se lancer dans cette entreprise audacieuse. Ganwan songeait d'abord à placer les futures cendres de son ancêtre de la cinquième génération, son richissime arrière-grandpère Guobao, dans une maison qu'il avait hérité de lui. Lorsqu'il alla voir les dirigeants du village pour justifier sa démarche et leur demander de le laisser faire, ceux-ci lui demandèrent si au lieu de penser à lui, il ne voulait pas faire quelque chose «pour l'ancêtre Zhenneng ». Il accepta et prit la tête de l'opération en posant trois conditions, dont la première était l'appel aux contributions financières des Chinois d'Outre-mer'. Une fois le mausolée construit, c'est en faisant jouer ses relations au sein de la sphère gouvernementale municipale qu'il a pu obtenir que la tombe de Zhenneng soit classée au patrimoine culturel (wenhua yichan). Selon ses propres mots, il était bien placé pour savoir que la politique du parti était changeante, et il avait donc souhaité assurer la protection éternelle de la tombe.

En cela, la stratégie adoptée par les Chen de Fort-les-Pins n'est pas très différente de celles d'autres lignages des alentours. Lors d'une visite au bureau de la culture et du patrimoine du district, j'ai en effet pu constater que la tombe de Zhenneng était placée sur la liste des 《sites patrimoniaux»(wenwudian 文物点) parmi plusieurs autres

9 Lesquels ont versé 1,5 millions de yuan sur un total de 2 millions collectés et utilisés pour le mausolée. 
tombes qui sont vraisemblablement celles d'ancêtres fondateurs d'autres lignages. Notons toutefois que l'édification d'un mausolée d'une telle ampleur constitue, à ma connaissance, un cas unique dans cette région du nord de Shenzhen. Les Chen de Fort-les-Pins se distinguent en effet d'autres lignages par leur démographie, et le grand nombre de parents dans la diaspora. Sans le soutien financier de ces derniers, l'édification du mausolée aurait été impossible.

Il me semble en outre important d'approfondir cette idée que la mobilisation collective pour l'ancêtre commun n'allait pas de soi. J'irais même jusqu'à avancer que l'objectif de réunir les cendres de tous les ancêtres dans un même endroit l'emportait sur la sauvegarde des ossements de Zhenneng. Il est difficile de prouver ce point. Néanmoins, un indice relativement probant est l'argument, avancé à plusieurs reprises aux cours des conversations avec les principaux acteurs du projet, que ce sont les Chen d'Outre-mer qui étaient les plus attachés à la sauvegarde de la tombe, pour des raisons tenant à ses propriétés géomantiques. En effet, me disait-on, le fengshui de la tombe de Zhenneng était censé agir au lointain et à l'extérieur, c'est-à-dire sur les femmes (qui se marient en principe à l'extérieur du lignage Chen, donc du village) et les émigrés ${ }^{10}$. Logiquement, ceux qui tenaient le plus à conserver en l'état la tombe de Zhenneng étaient donc ceux qui en bénéficiaient le plus, les Chen de Hong Kong et de la diaspora. Ayant assisté au rite collectif accompli pour Zhenneng en mars 2013, j'ai été frappée par la forte présence des Hong Kongais et Chinois d'Outre-mer « retournés » (guiqiao 归侨) au sein du petit groupe, comprenant une quinzaine de personnes, principalement des hommes, qui accomplit le rituel sous la direction du président hong-kongais de la fondation Zhenneng. Au cours des heures précédentes avait défilé devant la tombe un flux faible mais constant de personnes venues sacrifier à Zhenneng (ainsi que son épouse et ses fils) à titre individuel. Parmi ces personnes, la proportion de femmes était située entre deux tiers et trois quarts ${ }^{11}$.

Le culte rendu à l'ancêtre fondateur Zhenneng, s'il est organisé et financé par la fondation, n'est donc pas uniquement collectif ; il est tout autant une affaire individuelle. Comme l'a souligné Wolf (1976 : 346), il serait réducteur d'interpréter les rites communs comme l'expression mécanique d'une solidarité agnatique. C'est ce point que Maurice Freedman ne parvient pas à voir dans un chapitre où il analyse les questions de géomancie. Freedman commence par proposer de voir dans le fengshui des tombes une façon de chercher à distinguer sa fortune de celles des autres. Plus l'ancêtre est lointain, plus nombreux sont les collatéraux qui risquent d'en profiter; à l'inverse, plus il est proche, plus il promet de bénéficier à soi seul. « La patrilinéarité lie les fortunes des agnats ; la géomancie leur donne la chance d'individualiser leurs destins » (1966: 131). Il en vient ainsi à établir une distinction entre deux aspects du culte des ancêtres, l'aspect « vénération » (worship) et l'aspect " géomantique ». Le premier concerne le culte rendu au temple, via la tablette, et met l'accent sur l'unité et l'harmonie du groupe de descendance. Le second concerne la géomancie des tombes et implique individualisme et compétition. Ces deux aspects correspondent, avance

10 Les villageois disent aujourd'hui que le fengshui a changé, et leur est désormais favorable, mais je ne peux développer ce point dans l'espace de cet article.

11 Un autre indice est la moindre fréquentation du mausolée le jour de Chunfen par comparaison avec Qingming, jour du culte aux ancêtres proches. 
Freedman, à des modes d'apparition différents des ancêtres. Alors que sous son aspect tablette, l'ancêtre vénéré est actif et peut dispenser des bienfaits, sous son aspect « ossements ", l'ancêtre est un jouet passif aux mains de ses descendants qui peuvent à leur gré le manipuler en jouant sur la localisation de sa tombe.

Ces deux modes d'apparition différents renvoient donc selon Freedman à deux attitudes opposées vis-à-vis des ancêtres et des comportements différents entre agnats (unité ou compétition). Bien qu'il reconnaisse que les ancêtres font l'objet d'un culte sur leurs tombes et qu'il y a donc un certain recoupement entre géomancie et worship, il avance que le contraste entre les deux n'en reste pas moins valable. Ceci doit conduire, selon lui, à examiner de plus près le statut des ancêtres $(1966: 143)^{12}$.

\section{L'ancêtre fondateur comme unificateur du collectif}

Le contraste établi par Freedman entre les deux modes d'apparition des ancêtres et les modes de relation qui leur sont associés paraît valable pour différencier les deux types de culte, l'un au temple, l'autre sur les tombes. Freedman ne considère les ancêtres communs que sous leur aspect de tablette, sans tenir compte du fait qu'ils sont l'objet d'un culte unitaire sur leur tombe. Réciproquement, il envisage l'aspect tombal du culte comme relevant uniquement des ancêtres les plus proches. Or, en ce qui concerne l'ancêtre commun, cette distinction ne vaut pas.

J'en arrive ainsi à l'interprétation que je voudrais proposer. L'ancêtre commun est, non pas l'ancêtre de sous-groupes ou de segments du lignage, mais l'ancêtre de tous, c'est-à-dire du lignage dans sa totalité en même temps que de chaque individu descendant de lui. C'est en cela précisément qu'il fait le lien entre les deux opérations, celle de protection des ossements dans la tombe et celle de logement des cendres de tous les autres ancêtres. Il assure le lien en ce que, si tous ces ancêtres sont à titre individuel les ancêtres propres et immédiats des vivants, ils sont simultanément ses descendants. Voir ceci suppose un changement de perspective ; d'adopter le point de vue, surplombant, de Zhenneng plutôt que celui, " par en bas », des descendants. Il suppose de quitter une vision individuelle pour adopter celle du collectif. C'est ce que font les Chen de Fort-les-Pins lorsque, au lieu de parler comme ils le font souvent, de « leurs propres ancêtres » (ziji de zuxian), ils les désignent par l'expression « les descendants de Zhenneng » (Zhenneng de houdai 振能的后代). Zhenneng est l'ancêtre de tous mais aussi celui qui relie tous les ancêtres individuels. Si dans la première opération - celle de sauvetage de la tombe - le statut de l'ancêtre est celui de l'objet de culte, Zhenneng fait pour la seconde - celle de stockage des ancêtres - figure de rassembleur. Il est donc celui qui permet de transcender l'individualisme poussant chacun à se préoccuper d'abord de ses propres ancêtres.

C'est cette même idée qui se retrouve dans la manière dont, dans la vie courante (hors du contexte officiel de la généalogie, où ils se désignent comme les « Chen de Fort-les-Pins ») les membres du lignage s'y réfèrent par l'expression Zhenneng zu 振能族, lignage Zhenneng. Le nom de Zhenneng est donc une synecdoque - il fait

12 Parvenu à ce point, Freedman se lance dans comparaison avec la littérature africaniste concernant la distinction entre ancêtres collectifs et ancêtres individuels. Son empressement à rejeter les parallélismes avec la Chine relevés par Fortes (1953) semble lui avoir fait oublier le point de départ de sa réflexion. 
partie de l'ensemble qu'il vient à désigner. Zhenneng est un « individu collectif», non pas comme somme des composantes individuelles, mais en tant qu'il transcende les individualités dans une structure unique (Descombes 2001). Cela transparaît également dans l'expression employée pour décrire la manière dont Ganwan, le haut cadre, a été persuadé de prendre la tête du projet de protection : il lui a été demandé s'il ne voulait pas, plutôt que d'agir pour ses propres ancêtres, « faire quelque chose pour notre seigneur Zhenneng à tous » (women zhengge Zhenneng gong 我们整个振能公). Ceci impliquait d'entreprendre quelque chose non pas pour Zhenneng uniquement, mais pour tous les ancêtres.

Zhenneng incarne ainsi le «public»: si j'ai traduit gong 公 par « seigneur », dans la citation qui précède, titre honorifique par lequel un ancêtre fondateur est généralement désigné $^{13}$ (au moins chez les Hakka), gong signifie également « public ». Il entre dans la composition de l'expression gongyi 公益, le bien public. L'ancêtre fondateur permet le dépassement des intérêts privés pour le «bien public » et l'unification de la communauté lignagère et villageoise, comme en atteste la présence des autres patronymes dans le mausolée. Il pourrait s'apparenter, par sa geste fondatrice (la migration et l'installation à Fort-les-Pins) à la personne héroïque au sens que lui donne Marshall Sahlins (1985 : 47). L'un des traits caractéristiques du mode historique héroïque est l'emploi, par les chefs, du " je héroïque », qui narre à la première personne l'histoire du royaume tout entier. La comparaison s'arrête toutefois à ce phénomène de condensation de l'individu et du collectif, car ici c'est le collectif présent qui est fusionné dans l'ancêtre, et non le collectif passé qui se trouve condensé dans l'individu au présent ${ }^{14}$.

Ceci est encore conforté par la manière le nom de Zhenneng sert de bannière de ralliement. En témoigne la citation suivante extraite d'un entretien avec le vice-président de la fondation Zhenneng : « En utilisant le nom de Zhenneng (yong Zhennenggong de ming 用振能公的名) nous avons uni les compatriotes de Fort-les-Pins ici et à l'Outremer, pour la cause de Fort-les-Pins, pour sa construction économique, et pour le bien public (gongyi shiye). Nous avons incité des leaders à l'Outre-mer à mobiliser (les membres de leurs communautés), et tous, en entendant que quelque chose devait être fait pour Fort-les-Pins, pour l'ancêtre Zhenneng, ont répondu à l'appel comme un seul homme. " On voit là que Zhenneng est à la fois la cause de la mobilisation, et le nom qui incite à la mobilisation pour une cause qui en réalité le dépasse puisqu'elle concerne tous les ancêtres. Zhenneng n'opère donc pas simplement comme individu collectif, il est un opérateur d'unité.

Ce que je viens de souligner n'est pas en soi un phénomène nouveau. La fusion du collectif et de l'individuel dans la personne de l'ancêtre fondateur, et l'usage métaphorique du nom de l'ancêtre que cette condensation permet était à l'œuvre, dans les lignages d'avant 1949, dans la façon dont des terres étaient placées au nom de tel ou tel ancêtre commun. Les revenus de ces terres étaient destinés à son culte. David Faure

13 C'est également le cas parmi les Chinois hakka de Polynésie française en ce qui concerne Chim Soo Kung, malmort du temps des plantations dont le statut d' " ancêtre » ou de "divinité » fait d'ailleurs l'objet de débats au sein de la communauté (Trémon 2007).

14 Pour autant, cette inversion ne me semble pas relever de celle que met en avant Sahlins (1985) entre le mode héroïque de formation du lignage et le processus développemental du lignage segmentaire car précisément, ce n'est pas de segmentation qu'il s'agit ici. 
a souligné que c'est l'enregistrement de la propriété sous le nom de l'ancêtre qui, historiquement, permit l'émergence de l'organisation lignagère (2006 : 1303).

En revanche, la construction contemporaine du mausolée a eu un effet unificateur sur le village et le lignage en créant un second lieu de culte commun - le premier étant le temple ancestral. Là où auparavant le culte des ancêtres avait lieu sur les tombes dispersées autour du village, aujourd'hui toutes les familles se rendent au même endroit pour rendre leurs sacrifices aux ancêtres. Plusieurs personnes m'ont dit que c'était beaucoup plus pratique maintenant qu'il y a le mausolée, parce que lorsqu'elles n'ont pas le temps de revenir plusieurs fois pour sacrifier à des générations successives, elles viennent et « font » tous les ancêtres en une même journée. À Chunfen, le jour où a lieu le culte pour l'ancêtre fondateur Zhenneng, la construction du mausolée a permis que ce culte soit suivi d'un repas, comme c'est la tradition, mais un repas désormais pris en commun sur le site même du mausolée, au pied du monticule où se trouve la tombe. Dans le passé, il n'y avait pas d'installations permettant de cuisiner sur place et le site ne s'y prêtait guère. Le repas était cuisiné dans l'enceinte du temple ancestral et chacun prenait une portion qu'il ramenait à la maison pour le consommer en famille. Désormais, même au jour de l'anniversaire de Zhenneng, en octobre, le repas est pris au mausolée. Et à Qingming, le jour où l'on sacrifie à ses propres ancêtres, plusieurs familles s'étaient cotisées pour acheter de la nourriture, la cuire et la consommer en commun.

Le mausolée a eu un effet unificateur sur la communauté villageoise et lignagère, en créant un point de rassemblement, de tous les ancêtres, dont les tombes étaient dispersées, mais aussi des vivants, qui ont là une occasion supplémentaire de se retrouver $^{15}$. Il y a eu là aussi un processus de création d'un espace public, d'un lieu, défini par son point focal, de rassemblement et d'effervescence collective. Les Chen ont créé un site où ils pourront à leur tour être placés après leur mort, la place dans le mausolée étant gratuite pour les habitants du village - en cela aussi, le mausolée est présenté comme relevant du « bien public ». En outre, pour rendre leur projet plus conforme aux exigences des autorités, les villageois Chen ont cherché à faire apparaître ce site comme un site public (rappelons que la réforme funéraire exige que les urnes soient stockées dans des cimetières publics) en le renommant « Jardin du repos de Fort-les-Pins » (au lieu de « Jardin de la tombe de l'ancêtre fondateur Zhenneng »). À noter que ce terrain est demeuré propriété collective du village nouvellement urbanisé en 2004. En l'absence de mausolée, la terre où se trouve le site aurait probablement été réquisitionnée, alors qu'elle est encore aujourd'hui gérée par ce qui reste des anciens collectifs, la société par actions à l'échelon de l'ancien village.

\section{Conclusion}

Dans un contexte où les autorités de la Chine des réformes capitalistes s'emploient à éradiquer ce que le maoïsme n'avait pas réussi à supprimer, les membres du lignage se sont réunis pour défendre leur plus petit dénominateur commun, l'ancêtre fondateur.

15 On pourrait même avancer, avec les précautions nécessaires étant donné que ce n'est pas un sujet que les gens de Fort-les-Pins abordent aisément, que tous les ancêtres partagent aujourd'hui la géomancie de la tombe de Zhenneng. Il y aurait en quelque sorte une collectivisation géomantique. 
Fragile et menacée, l'ancestralité a mué pour se réduire, en quelque sorte, à l'essentiel. Alors que certains auteurs voient dans la religion populaire l'affirmation d'une sphère morale opposée à celle de l'État (Feuchtwang 2000 : 173 ; Yang 2004) ou assimilent les stratégies déployées par les acteurs de ces cultes à une recherche de «légitimation» vis-à-vis de l'État (Chau 2011 : 6 ; Flower 2004 : 677), le culte lignager participe de l'ensemble des affaires « publiques » que le lignage a pour vocation de conduire, et en cela, il se déploie en parallèle à l'État plutôt qu'en opposition à celui-ci. Même dans un contexte comme celui de la réforme funéraire où la logique lignagère se retrouve en porte-à-faux par rapport aux objectifs poursuivis par l'État, l'action collective des membres de la communauté lignagère n'est pas conçue comme illégale - ce qui prévaut, nous l'avons vu, est une recherche de légalité (plus que de légitimité).

Dans un climat idéologique où les conceptions de l'ancestralité ne peuvent être affichées ouvertement, et où les autorités s'en prennent directement à ce qui est considéré comme relevant de superstitions, l'action entreprise par les membres du lignage Chen permet de dégager ce à quoi ils tiennent le plus. La protection de ses restes supposait cependant une coordination collective qui n'est pas allée de soi. Le moment de flottement qui s'est produit dans les mois suivant l'annonce de la réforme funéraire révèle que le premier réflexe était plutôt de s'occuper de " ses propres ancêtres ». Cette dualité ancêtre fondateur/ancêtres proches, qui trouve à s'exprimer en temps normal à travers les deux formes de culte ancestral, devait ici être surmontée en temps de crise. La solution qui a été trouvée, celle de loger les cendres des ancêtres proches aux côtés de l'ancêtre fondateur, permet de préciser le statut de ce dernier. Il apparaît comme un opérateur du collectif et un principe public ; ancêtre de chacun pris individuellement, il est aussi l'ancêtre du corps social lignager-villageois dans son ensemble. Agir pour lui est aussi agir en son nom pour le bien de tous. Il est, au cours de ce processus, le but de l'opération de sauvegarde, mais il est dans le même temps la cause commune, celle du bien public, pour laquelle tous se mobilisent afin que chacun trouve une solution assurant le repos à ses propres ancêtres. En somme, cela a supposé que les membres de certaines branches ayant connu une réussite sociale et tournés vers leurs ancêtres les plus éminents, œuvrent pour tout le monde. On assiste ainsi à une forme de désegmentation du lignage.

L'ancestralité s'en est trouvée transformée, dans le sens où l'ancêtre fondateur est désormais un passage obligé pour tous ceux qui se rendent au mausolée. Si, comme par le passé, le rite collectif en tant que tel n'implique pas tout le monde, tous passent désormais devant sa tombe lorsqu'ils vont s'occuper de leurs propres ancêtres. L'accentuation de la place de l'ancêtre fondateur était déjà en cours durant les décennies précédentes, dès lors que c'était lui qui reliait l'ensemble de ceux qui s'étaient réfugiés à Hong Kong - en témoigne l'établissement de la fondation à son nom.

Enfin, Zhenneng est celui qui assure la continuité de l'identité lignagère, mais aussi villageoise, là où le village a disparu physiquement et légalement. On peut y voir, dans le contexte de l'afflux de migrants travailleurs (même si ceux-ci sont dépourvus de droits de résidence) une forme d'affirmation de l'appartenance à la communauté des villageois autochtones, yuancunmin, autour de ce point focal qu'est la tombe de l'ancêtre fondateur du village et du lignage. La destruction de sa tombe aurait signifié l'effacement du dernier point de repère territorial par rapport auquel se situent les anciens villageois, tant ceux qui sont restés que ceux qui sont partis. L'ancestralité 
assure le maintien d'un point de référence ancré territorialement dans un contexte de mutations accélérées, en même temps qu'elle est reformulée dans le nouveau cadre urbain. Alors que le nom de Fort-les-Pins a disparu en même temps que le village, celui de l'ancêtre perpétue la référence. Le temple ancestral et le mausolée, mais aussi l'école élémentaire Zhenneng et le lycée, ainsi que "l'immeuble de la culture Zhenneng », constituent des marqueurs visibles dans le paysage radicalement transformé. L'usage symbolique de son nom ne se substitue pas, cependant, à la présence perpétuelle de l'ancêtre dans sa tombe.

\section{Références citées}

Ahern, Emily, 1973. The Cult of the Dead in a Chinese Village. Stanford : Stanford University Press.

Aijmer, G., 1967. « Expansion and Extension in Hakka Society », Journal of the Hong Kong Branch of the Royal Asiatic Society, 7, pp. 42-49.

Baptandier, Brigitte (éd.), 2001. De la malemort dans quelques pays d'Asie. Paris : Karthala.

-, 2012. « Du meurtre symbolique du père et de l'aspect insaisissable du présent », Extrême-Orient, Extrême Occident, hors série, pp. 277-311.

BrandtSTÄDTER, Susanne, (2013). Counterpolitics of Liberation in Contemporary China: Corruption, Law, and Popular Religion, Ethnos: Journal of Anthropology, 78 (3), pp. 328-351.

CHAU, Adam Yuet, 2011. «Introduction : Revitalizing and Innovating Religious Traditions in Contemporary China ", in Adam Yuet Chau (éd.), Religion in Contemporary China : Revitalization and Innovation, pp. 1-31. Londres : Routledge.

Chun, Allen, 1996. "The Lineage-Village Complex in Southeastern China. A Long Footnote in the Anthropology of Kinship », Current Anthropology, 37 (3), pp. 429-450.

Cohen, Myron L, 1969. «Agnatic Kinship in South Taiwan », Ethnology, 8, pp. 167-182.

-, 1996. « The Hakka or 'Guest People', Dialect as a Sociocultural Variable in Southeast China », in Nicole Constable (éd.), Guest People, Hakka Identity in China and Abroad, pp. 36-79. Seattle : University of Washington Press.

Dean, Kenneth, 2003. " Local Communal Religion in Contemporary South-east China ", in Daniel L. Overmyer (éd.), Religion in China Today, pp. 32-52. The China Quarterly, Special Issues, New Series, 3.

Descombes, Vincent, 2001. «Les individus collectifs », La Découverte, Revue du MAUSS, 2 (18), pp. 305337.

Ebrey, Patricia, 1986. «The Early Stages in the Development of Descent Group Organization », in Patricia Ebrey et James Watson (éds), Kinship Organization in Late Imperial China, 1000-1940, pp. 16-61. Berkeley : University of California Press.

FAure, David, 1989. « The Lineage as a Cultural Invention », Modern China, 15 (1), pp. 4-36.

-, 2006. « La solution lignagère. La révolution rituelle du $16^{\text {ème }}$ siècle et 1'État impérial chinois », Annales, Histoire, Sciences Sociales, 6, pp. 1291-1316.

Feuchtwang, Stephan, 2000. « Religion as Resistance », in Elizabeth J. Perry et Mark Selden (éds), Chinese Society, pp. 161-77. Stanford : Stanford University Press.

Flower, John M., 2004. «A Road is Made : Roads, Temples, and Historical Memory in Ya'an County, Sichuan », Journal of Asian Studies, 63 (3), pp. 649-85.

Fortes, Meyer, 1953. «The Structure of Unilineal Descent Groups », American Anthropologist, 55 (1), pp. 17-41. 
Freedman, Maurice (éd.), 1958. Lineage Organization in Southeastern China. Londres : Athlone Press.

-, 1966. Chinese lineage and society : Fukien and Kwangtung. Londres : Athlone Press.

-, 1970. Family and Kinship in Chinese Society. Stanford : Stanford University Press.

Gates, Hill, 1987. « Money for the Gods », Modern China, 13 (3), pp. 259-277.

Harrell, Steven, 1974. "When a Ghost Becomes a God », in A. P. Wolf (dir.), Religion and Ritual in Chinese Society, pp. 193-206. Stanford : Stanford University Press.

JING, Jun. 1996. The Temple of Memories : History, Power, and Morality in a Chinese Village. Stanford : Stanford University Press.

Johnson, Jonathan Carl, 2011. Reforming the Dead : The Intersection of Socialist Merit and Agnatic Descent in a Chinese Funeral Home. Thèse de doctorat, University of California, Los Angeles. ProQuest, UMI Dissertation Publishing.

Jordan, David K., 1972. Gods, Ghosts, and Ancestors : the Folk Religion of a Taiwanese Village. Berkeley : University of California Press.

Lagerwey, John, 1997. «Préface », in Fang Xuejia (éd.), Meizhou Heyuan Diqu de Cunluo Wenhua. (Culture villageoise territoriale dans le Nord-est du Guangdong). Hong Kong : Association internationale d'Études Hakka et École française d'Extrême-Orient.

LeOng, Sow-Theng (éd. par T. Wright (posth.)), 1997. Migration and Ethnicity in Chinese History, Hakkas, Pengmin, and their neighbors. Stanford : Stanford University Press.

LewIS, Mark Edward, 2006. The Construction of Space in Early China. Albany : State University of New York Press.

Po, Lanchih, 2012. «Asymmetrical Integration : Public Finance Deprivation in China’s Urbanized Villages », Environment and Planning, 1 (44), pp. 2834-51.

SAHLIns, Marshall, 1985. Islands of History. Chicago : University of Chicago Press.

SiU, Helen, 1989. " Recycling Rituals : Religion and Popular Culture in Contemporary Rural China », in P. Link, R. Madsen et P. G. Pickorowicz (éds), Unofficial China : Popular Culture and Thought in the People's Republic, pp. 121-137. Boulder : Westview Press.

TRÉMON, Anne-Christine, 2007. « Mémoire d'immigrés et malemort», dossier « Patrimoine et immigration », Cahiers du FRAMESPA (en ligne <http://w3.framespa.univ-tlse2.fr/revue/articles_fiche. php?id=263>).

WANG, Ya Ping, Yanglin WANG et Jiansheng Wu, 2009. «Urbanization and Informal Development in China : Urban Villages in Shenzhen ", International Journal of Urban and Regional Research, 22 (4), pp. 957-73.

Watson, James, 1975. Emigration and the Chinese Lineage: The Mans in Hong Kong and London. Berkeley : University of California Press.

-, 1988. «Funeral Specialists in Cantonese Society : Pollution, Performance, and Social Hierarchy », in James L. Watson et Evelyn Rawski (éds), Death Ritual in Late Imperial China, pp. 109-134. Berkeley : University of California Press.

Watson, James, Yu-bin Shen, Jin ShaO, 1982. « Of Flesh and Bones : The Management of Death Pollution in Cantonese Society », Journal of Guanxi University for Nationalities, 20 (6), pp. 38-49.

Whyte, M. K., 1998. " Death in the People's Republic of China », in James L. Watson et Evelyn S. Rawski, (eds), Death Ritual in Late Imperial and Modern China, pp. 289-316. Berkeley : University of California Press.

Wolf, Arthur P., 1974. « Gods, Ghosts and Ancestors », in Arthur P. Wolf (éd.), Religion and Ritual in Chinese Society, pp. 131-182. Stanford : Stanford University Press. 
-, 1976. «Aspects of Ancestor Worship in Northern Taiwan », in William H. Newell (éd.), Ancestors, pp. 339364. La Haye : Mouton.

YANG, Mayfair Mei-hui, 2000. « Putting Global Capitalism in its Place. Economic Hybridity, Bataille, and Ritual Expenditure », Current Anthropology, 41 (4), pp. 477-95.

-, 2004. « Spatial Struggles : Postcolonial Complex, State Disenchantment, and Popular Reappropriation of Space in Rural Southeast China », Journal of Asian Studies, 63 (3), pp. 719-55. 